\title{
Roles of the Interphase Stiffness and Percolation on the Behavior of Solid Propellants
}

\author{
Paul-Aymé Toulemonde, ${ }^{*[a, b]}$ Julie Diani, ${ }^{[c]}$ Pierre Gilormini, ${ }^{[a]}$ Geneviève Lacroix, ${ }^{[b]}$ and Nancy Desgardin ${ }^{[b]}$
}

\begin{abstract}
Atomic force microscopy has provided access to local moduli for propellants prepared with bonding agents, which create a stiffness gradient in the matrix producing a stiffer interphase surrounding the fillers. The reinforcing impact of the bonding agent appears up to some distance and interphase percolation is observed. In order to better understand the impact of bonding agents on the stress and strain at break of propellants, finite element simula-
\end{abstract}

tions are performed. Two-dimensional periodic cells containing randomly dispersed particles are considered, including both a cohesive zone model at the filler/matrix interface to account for possible debonding and an interphase that percolates or not. The influence of the interphase stiffness and of its percolation, on the stress and strain at break of the model propellants are evaluated through the use of a microstructure-based failure criterion.

Keywords: Propellants - Matrix/particle interface · Interphase · Mechanical behavior · Finite element analysis

\section{Introduction}

Composite solid propellants are elastomers highly filled with explosive particles (up to $90 \mathrm{wt}-\%$ ) [1]. The primary function of this material is to burn and deliver a phenomenal thrust for a very limited period. However, to ensure predictable burning kinetics and proper integration into industrial applications, specific stress and strain at failure are targeted. In this respect, the use of matrix/filler bonding agents has drawn significant attention. Mixed to the matrix, the bonding agent migrates to the filler surrounding and reacts in presence of filler or matrix, the latter reaction strengthening the filler/matrix bonds. Variants of this technique have been patented [2-4] for two of the main chemical systems of propellants: hydroxyl-terminated polybutadiene (HTPB) filled with ammonium perchlorate (AP) and glycidyl azide polymer (GAP) or polyethylene glycol (PEG) filled with organic particles. Looking closely at the experimental sections supporting these patents claims, one notes that adding some bonding agents in propellants enhances the stress at break significantly for HTPB/AP systems $[2,3,5]$ and for systems with organic fillers $[6,7]$. As regards the strain at break, the presence of a bonding agent can either reduce or improve it, changes ranging from $-25 \%$ to $+150 \%$ for HTPB/AP propellants and from $-58 \%$ to $+25 \%$ for systems with organic fillers are reported by the same authors. As a result, it appears that adding a filler/matrix bonding agent in a propellant can have three distinct beneficial effects on the failure properties of propellants: (i) improve the strain at break while reducing the failure stress, (ii) improve the stress without reducing the strain at break, and (iii) improve both properties at the same time.

Varying matrix/filler interface properties has demonstrated that the two first cases can be explained by changes of interface properties [8]. Such strengthening of the matrix/ filler interface is conventionally targeted by propellants manufacturers. However, until now, the modification of the interface properties only does not seem sufficient to explain the increase of both failure stress and failure strain at the same time. That is why another phenomenon has been proposed [6] to understand the effect of bonding agents: the appearance of a matrix stiffness gradient around the particles. An increase of the initial Young modulus is observed for systems where bonding agents enhance both failure properties [6]. This increase could stem from a local enhancement of the matrix stiffness due to an interphase created by the bonding agent.

The presence of an interphase at the filler/matrix interface has been accounted for in a range of composites. AFM nano-indentation has proved to be a valuable technique to characterize both the morphology and the mechanical properties of this interphase $[9,10]$ through the use of con-

[a] P.-A. Toulemonde, P. Gilormini Laboratoire PIMM, ENSAM, CNRS, CNAM 151 Boulevard de l'Hôpital 75013 Paris, France *e-mail: paul-ayme.toulemonde@ensam.eu

[b] P.-A. Toulemonde, G. Lacroix, N. Desgardin Herakles groupe Safran Centre de Recherche du Bouchet 9 Rue Lavoisier 91710 Vert-le-Petit, France

[c] J. Diani LMS, Ecole Polytechnique CNRS, Université Paris-Saclay 91128 Palaiseau, France 
tact mechanics [11]. It has already been used to reveal the presence of matrix/filler interphases in nanofiller [12] and microfiller [13] composites. This technique is also successful for soft materials [14]. Micro-mechanical models based on homogeneization theories [15] and FEA analysis [10,15-17] have been proposed to evaluate the influence of the characterized interphase on composites macroscale mechanical properties. However, these contributions focus on composites with a filler volume fraction of maximum $30 \%$, whereas propellants typically encompass over $70 \%$ fillers volume fraction. Considering that the amount and thus the effect of an interphase are directly related to the fillers specific surface, a full new study of propellants interphase influence on the mechanical behavior is required.

In the presented contribution, original experimental evidence of the existence of the matrix/filler interphase is shown and key characteristics of this interphase are identified. Next, 2D finite element simulations of a model material represented by a periodic cell of randomly dispersed fillers surrounded by an interphase and embedded in a rubber matrix are presented. Finally, a qualitative study of the effects of the interphase parameters on the mechanical response and failure of the composite is conducted. It provides an explanation for the possible effects of bonding agents on solid propellants.

\section{Experimental Section}

In this work, the material of interest is a Butalane ${ }^{\mathrm{TM}}$ solid propellant such as produced by Herakles. It consists of a compound of $68 \mathrm{wt} \%$ of ammonium perchlorate, $20 \mathrm{wt} \%$ of aluminum, and $12 \mathrm{wt} \%$ of a modified PBHT-based matrix that were mechanically mixed and crosslinked. In order to avoid surface roughness that would hinder AFM measurements, smooth surfaces were ultramicrotomed at $-70^{\circ} \mathrm{C}$ with a glass/diamond knife. The AFM measurement were carried out by BiophyResearch company with a Bruker Multipode 8 NanoscopeV $^{\mathrm{TM}}$ machine using Peak-force QNM $^{\mathrm{TM}}$ for peak-force tapping mode with scanasyst-Air ${ }^{\mathrm{TM}}$ probes with stiffness $12 \mathrm{~N} \mathrm{~m}^{-1}$.

Figure 1 and Figure 2 present the local map of the recorded Young modulus on Butalane ${ }^{\mathrm{TM}}$ samples. Three main phases appear on these images: (i) the matrix in dark grey, (ii) the filler particles in white, and (iii) an interphase surrounding the particles in light grey. In Figure 1, a particle with a radius seemingly over $30 \mu \mathrm{m}$ and particles much smaller are observed. At this scale, it appears that the interphase is located around the particles and spread over the microstructure. It also appears that the interphase thickness varies from one particle to another. In some cases, the interphase thickness is as large as the surrounded particle radius and in other cases it is difficult to acknowledge the presence of such an interphase.

Looking closely at a couple of small particles on Figure $2 b$, it is noted that the interphase encircles all the parti-

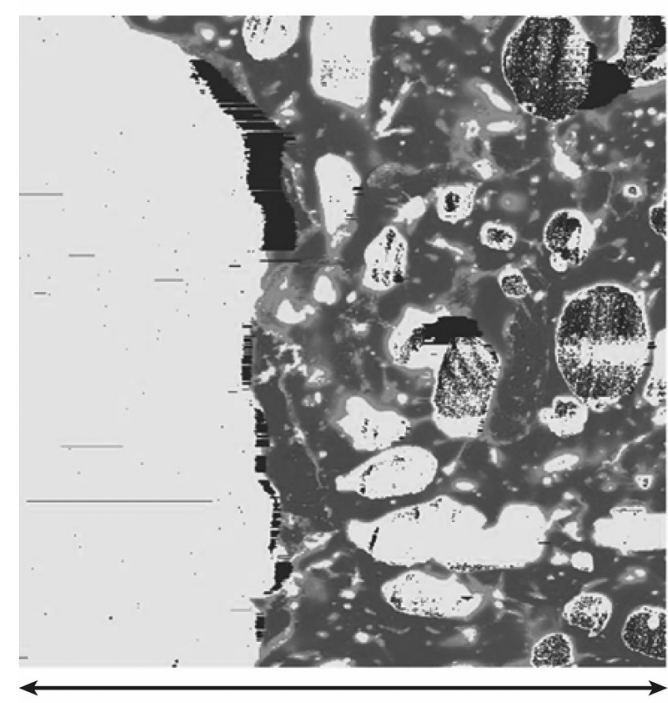

$30 \mu \mathrm{m}$

Figure 1. AFM observations of a portion of a large particle surrounded by smaller particles in Butalane ${ }^{\mathrm{TM}}$.

(a)

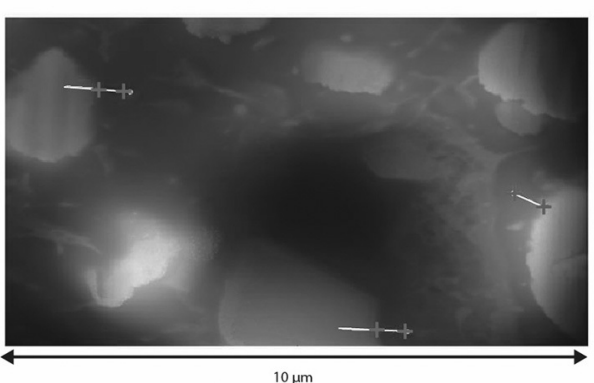

(b)

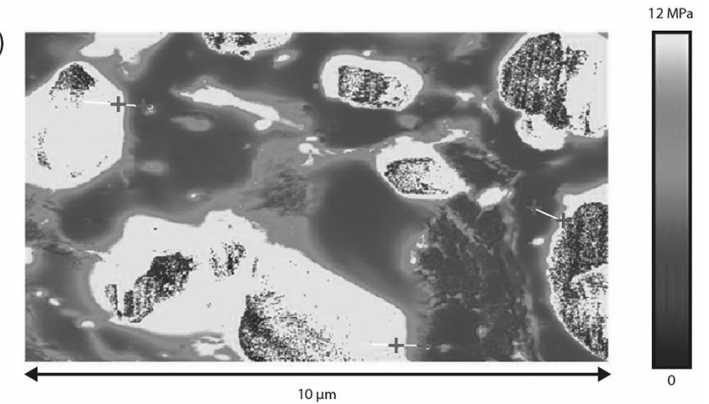

(c)

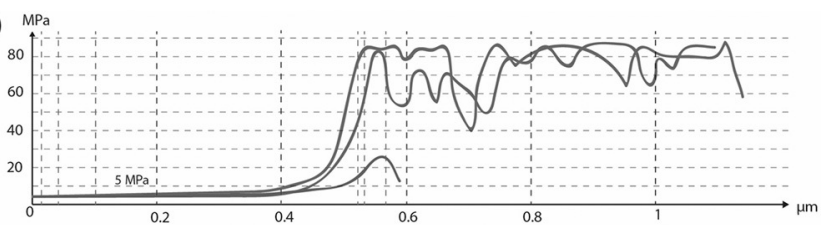

Figure 2. AFM observations of a couple of small particles: (a) topological image, (b) Young modulus map, and (c) evolution of the phases stiffness along the matrix/filler interphase in three different locations. 
cles to create a shell. This figure also emphasizes that contact points exist between these shells. Consequently, due to the high specific surface of fillers enhanced by the presence of small particles into the material, the interphase may percolate through the microstructure.

Figure $2 c$ presents the evolution of the Young modulus through matrix/filler interphases in three different locations illustrated on Figure $2 a$ and $b$. The local values of the phases stiffness are thus measured and it appears that: (i) the matrix displays a Young modulus of $5 \mathrm{MPa}$, (ii) the stiffness of the particles is too high to be measured with the probe, and (iii) the interphase is approximately five times stiffer than the matrix.

\section{Numerical Simulations}

Experimental evidences of filler/matrix debonding have been obtained on model [5] and industrial highly filled elastomers $[18,19]$. This damaging process plays a key role in the mechanical response of these materials in unixial tension [20]. Modeling efforts were conducted recently in order to understand and predict the behavior of these composites. In order to account for the filler/matrix debonding, a cohesive zone model was introduced to represent the filler/matrix damageable interface. Micromechanical modeling was performed [21,22] to account for small strain behaviors with large amounts of fillers or for hyperelastic behaviors with moderate amounts of fillers [23]. Some authors also carried out two-dimensional and threedimensional finite element analysis. The numerical feasibility of these calculations was assessed in the literature [2426]. Insight was gained on the effect of filler size on the mechanical behavior of composites described by a periodic lattice of particles [27] or by randomly dispersed particles [8]. The effect of the cohesive zone parameters on the mechanical response was studied [28] and comparisons with multiscale modeling were proposed $[22,29]$. The presence of an interphase around the particles was also accounted for in the case of elastomers filled up to a $26 \%$ volume fraction of fillers [17].

In this work, since our interest focuses on the behavior up to failure of propellants, microstructures containing a high volume fraction of fillers are submitted to large strains. In order to detect damage localization, randomly dispersed fillers are considered. A cohesive zone model is also introduced to model the damageable filler/matrix interface. In order to account for the matrix stiffness gradient around particles, layers of interphase that may percolate or not are added. To reduce the complexity of the calculations, two-dimensional simulations are considered here. They will provide qualitative comparisons with experiments and will allow testing more parameters due to the reduced computational cost. First, we present the microstructures, next the material and interface models, and finally the implementation in the Abaqus/Standard [30] finite element code.

\section{Microstructure}

It has been demonstrated $[8,27,28]$ that the presence of small particles mainly strengthen the matrix without noticeably influencing the composite failure properties in the case of a propellant with a large distribution of particles size. As outlined in the Experimental Section, it also appears that in the presence of an interphase, the small particles promote the percolation of the interphase through the microstructure thanks to their high specific surface. For these reasons, the propellant microstructure is represented by $2 \mathrm{D}$ periodic cells containing distributions of 49 round particles with a single particle size representing only the large particles and a given particle surface fraction of $50 \%$. In order to build the cell, the procedure presented in reference [31] is applied. A layer of interphase of relative thickness $e$ (evaluated as a fraction of the particle radius) is added around each particle. Figure 3 shows that, depending on the interphase relative thickness $e$, overlapping of the particle interphase may conduct to percolation of the interphase (Figure 3a) or not (Figure 3b).

Due to expected numerical behavior and failure scattering, four random microstructures are created and tested for each set of parameters considered.

\section{Material and Interface Model}

The matrix is considered as quasi-incompressible and hyperelastic and defined by its strain energy density $W$. In order to reduce the complexity of the numerical model a compressible neo-Hookean law is chosen $\left(W=E_{\mathrm{m}}\right.$ / $6\left(I_{1}-3\right)+K_{\mathrm{m}} / 2\left(\Delta V / V_{0}-1\right)^{2}, l_{1}$ being the first strain invariant). The matrix behavior is defined by its small strain Young modulus $E_{\mathrm{m}}$ and its bulk modulus $K_{\mathrm{m}}$. It has been demonstrated that such model yields results that are consistent with propellants mechanical behaviors in term of stressstrain relation and failure properties [8].

Despite the lack of direct evidence it can be assumed safely that the interphase is also a quasi-incompressible and hyperelastic material with a gradient of stiffness through its thickness, which may be averaged to model its behavior by a compressible neo-Hookean law with a Young modulus $E_{\mathrm{i}}$ (respectively a bulk modulus $K_{\mathrm{j}}$ ) larger than $E_{\mathrm{m}}$ (respectively $K_{\mathrm{m}}$ ). The ratio $H$ of interphase Young modulus over matrix Young modulus is therefore equal to $H=E_{\mathrm{i}} / E_{\mathrm{m}}$.

The particles are regarded as rigid.

The microstructures contain two kinds of interfaces: a matrix/interphase interface and an interphase/filler interface. Considering the chemical similarity of the interphase/ matrix pair, this interface is regarded as perfectly bonded and thus undamageable. As explained above, debonding around filler particles is a key feature to understand the mechanical response of propellants up to failure. Therefore, a cohesive zone model is introduced at the interphase/filler interface to account for this debonding. This model represents the interface through an elastic-damageable traction- 
(a)

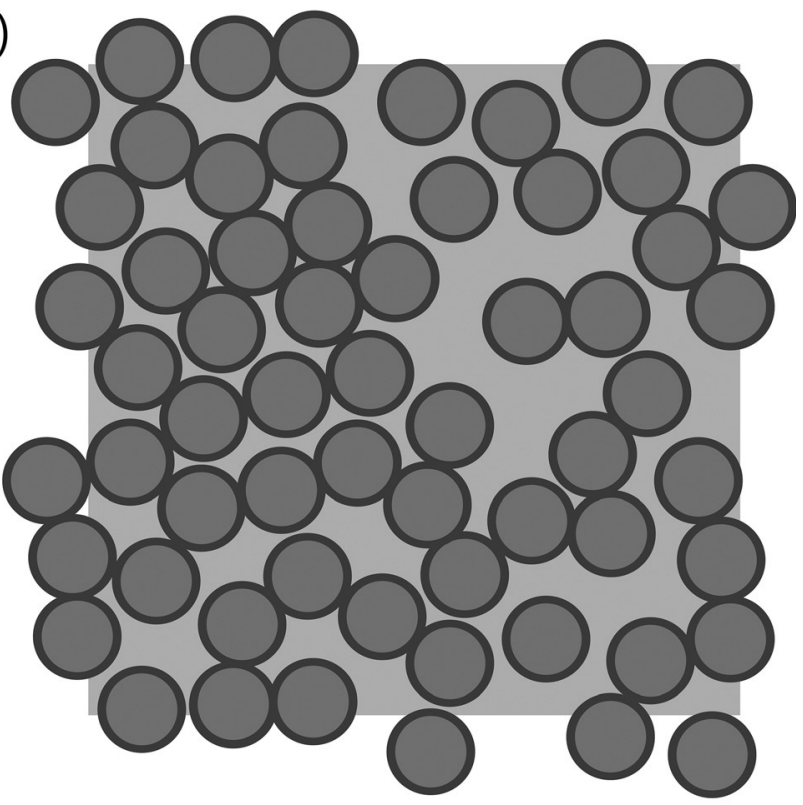

$\begin{array}{llll}\text { Matrix } & \text { Interphase } \quad \text { Filler }\end{array}$

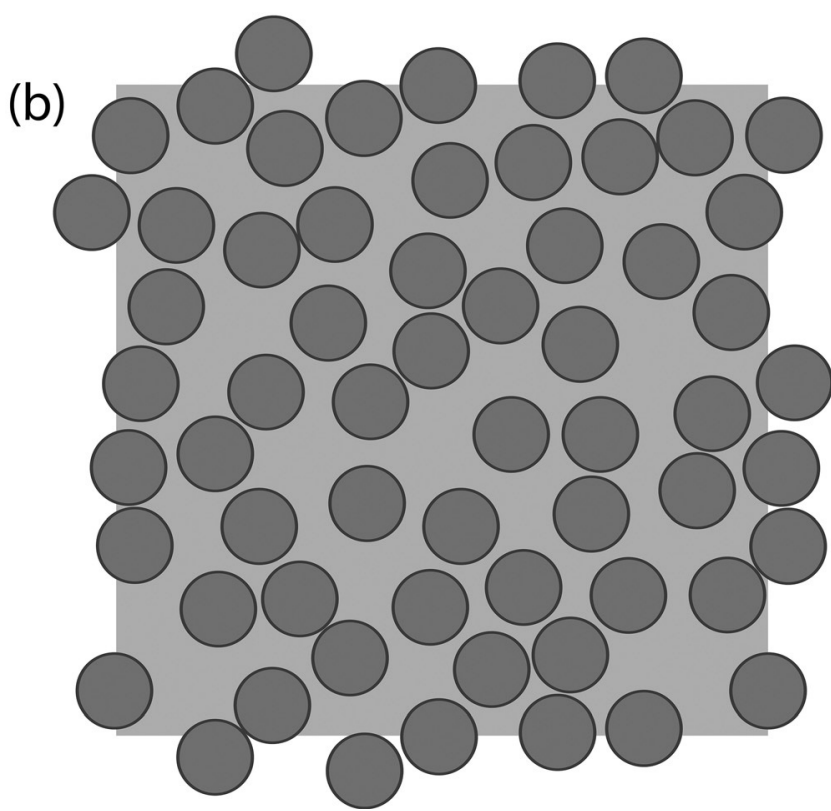

Figure 3. Modelled microstructures: (a) with interphase percolation (e=0.12), (b) without interphase percolation $(e=0.035)$.

separation law. Park and Paulino [32] have reviewed multiple variants of the cohesive zone models that account for a range of phenomena linked to decohesion (damage behavior, mode-mixing, in 3D especially). Among the models reviewed, a bilinear traction-separation law deriving from a potential energy independent of mode-mixing and ensuring the same fracture energy for any loading path has been proposed [33]. Its simplest variant is illustrated in

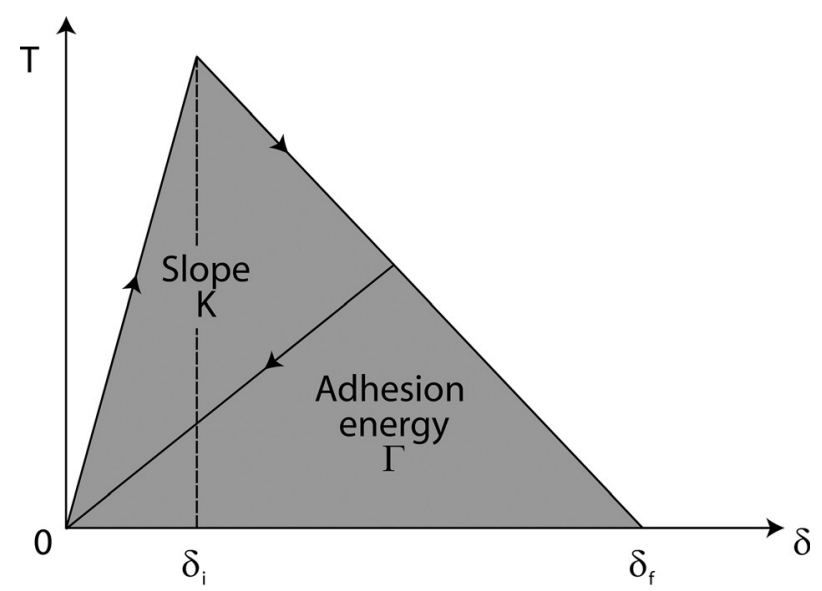

Figure 4. Illustration of the chosen cohesive zone model for a positive normal traction applied to the interface.

Figure 4 and it is described by four parameters: $K$ (initial "pseudo-rigidity"), $\Gamma$ (adhesion energy), $\delta_{\mathrm{i}}$ (critical displacement for damage initiation), and $\delta_{f}$ (critical displacement for interface failure). Only three of these parameters are independent since they are related by $\Gamma=K \delta_{\mathrm{i}} \delta_{f} / 2$. Furthermore, to model the fact that no debonding is allowed before the appearance of the interface damage, $K$ is chosen as high as computation allows. Thus the chosen cohesive zone model is defined by two parameters: $\delta_{f}$ and $\Gamma$.

\section{Implementation}

The simulations are run with Abaqus/Standard [30] using 4node hybrid plane strain elements with reduced integration. Periodic displacement boundary conditions were applied to the cell. So as to ensure a ratio of 3 between the critical length $\delta_{f}$ of the cohesive zone model and the element size [34], the structures were meshed with an average of 300000 elements. It was verified on a periodic lattice of particles with the same microstructural and material parameters that for the chosen mesh size or lower mesh sizes, the mechanical behavior is independent of the element size.

The particles radius is $0.1 \mathrm{~mm}$, which is in the range of common solid propellants fillers radii for large particles $[25,26,35]$. The filler surface fraction is $50 \%$, which suitably represents the volume fraction of large particles in standard solid propellants [36]. The neo-Hookean stiffness of the propellants matrix is $E_{\mathrm{m}}=E_{\mathrm{m}}{ }^{0}=5 \mathrm{MPa}$ as was evaluated in AFM measurements on Figure 2 and remains fixed, whereas the interphase behavior is given as $E_{\mathrm{i}}=H E_{\mathrm{m}}$ with $H=2$ or 5 or 10. These values of $H$ provide a realistic range of values according to the AFM measurements. Values of $K_{\mathrm{m}}=$ $4000 \mathrm{MPa}$ and $K_{\mathrm{i}}=H K_{\mathrm{m}}$ were chosen to obtain a quasi-incompressible behavior. The parameters of the cohesive zone model are taken from the literature $[35,37]$ to represent a matrix/filler interface of energetic materials: $K=$ $1000 \mathrm{MPa}, \delta_{\mathrm{f}}=0.1 \mathrm{~mm}$ and $\Gamma=0.083 \mathrm{MPa} \mathrm{mm}$. Relative 
thickness $e$ values were chosen in order to experience possible percolation of the interphase through the microstructure, as suggested by AFM observations. Values $e=0.01$ and 0.035 do not produce percolation, whereas $e=0.1$ and 0.12 do.

\section{Results and Discussion}

\section{Failure Criterion}

Rivlin and Thomas [38] introduced a critical elastic energy density based failure criterion for elastomers. This criterion is a mere application of fracture mechanics and is consequently widely used. In the presented case, the underlying principle that failure initiates in material elements with high elastic energy density is extended. Indeed, starting from a virgin microstructure (Figure 5a), early damage of the interphase/filler interface initiates homogeneously throughout the microstructure (Figure 5b). At some point, debonding localizes orthogonally to the loading direction (Figure 5c), and interphase-matrix fibrils form highlighting very high levels of elastic energy density. Therefore the strain energy based failure criterion may be equivalently defined by the appearance of the fibrillar microstructure due to debonding localization. From a practical point of view, as this localized debonding occurs, the two remaining parts of the microstructure move as rigid bodies. Experimental evidence attests of such a localized failure mechanism [39].

The elastic energy density $W$ normalized by the smallstrain Young modulus $E_{\mathrm{m}}$ is a dimensionless measure of the elastic energy density and allows comparisons between simulations with different material parameters. Figure 6 illustrates the case, where no debonding localization occurs and the proposed failure criterion does not apply, compared to Figure 5c, where localization occurs. At $36 \%$ macroscopic strain, the no-localization microstructure does not display peaks of normalized elastic energy (Figure 6) in contrast with the case of debonding localization (Figure $5 c$ ).

Figure 7 presents the results of four identical simulations except for the particles random layout. Very good reproducibility is achieved on the behavior, while expected scattering is observed on failure. Therefore, average behaviors are presented in what follows with mean values and standard deviations of the strain at break. As regards the comparison to experimental data on propellants $[7,40]$, it appears that the shape of the stress-strain curve is well reproduced on Figure 7: first a linear portion is obtained, then the structure undergoes softening and a plateau is observed. Also, the range of strain at which softening begins and the failure strain are consistent. The order of magnitude of the simulated stress is consistent with propellants behavior at low temperature (typically $-40^{\circ} \mathrm{C}$ ) and overestimates propellants stress-strain response at room temperature. (a)

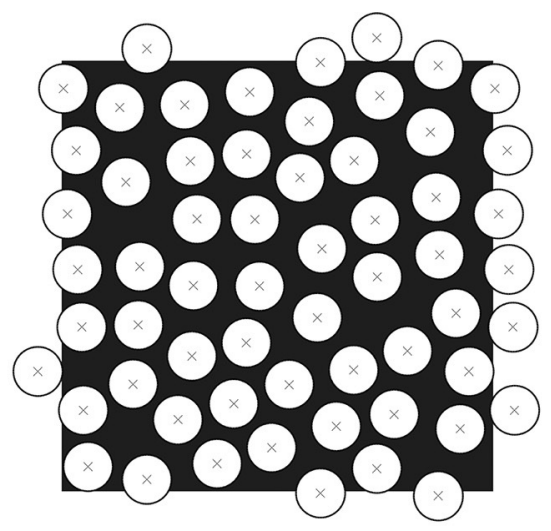

(b)

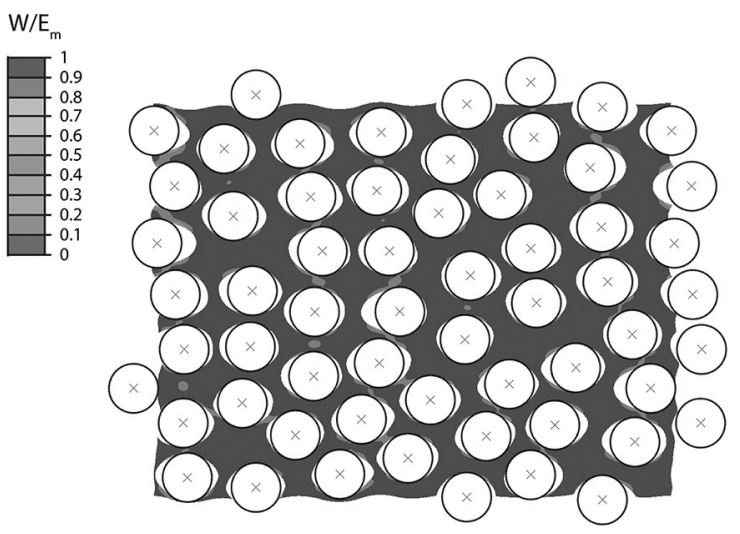

(c)
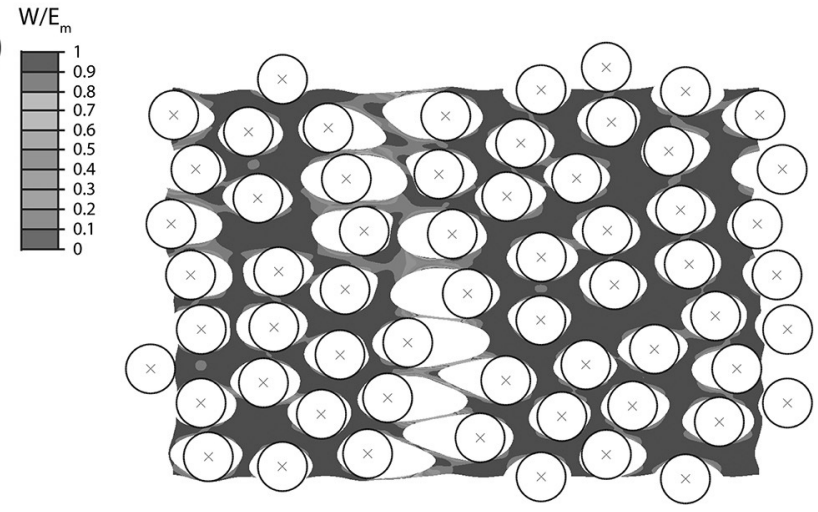

Figure 5. Evolution and ratio of the elastic energy density $W$ normalized by Young modulus $E_{\mathrm{m}}$ for a microstructure without interphase submitted to uniaxial tension. (a) Initial microstructure, (b) early damage (20\% macroscopic strain), and (c) further damage evolution with the appearance of matrix fibrils (36\% macroscopic strain) $\left(E_{\mathrm{m}}=E_{\mathrm{m}}{ }^{0}\right)$.

\section{Effect of the Interphase Thickness}

Figure 8 shows the impact of the interphase relative thickness $e$ on the mechanical behavior of the composite until failure with $H=5$ (when the interphase is five times stiffer than the matrix). As reference, the behavior of model materials with no interphase is also represented. First, at small strain, before any interface debonding has occurred, the stiffness of the structure increases with the relative thick- 


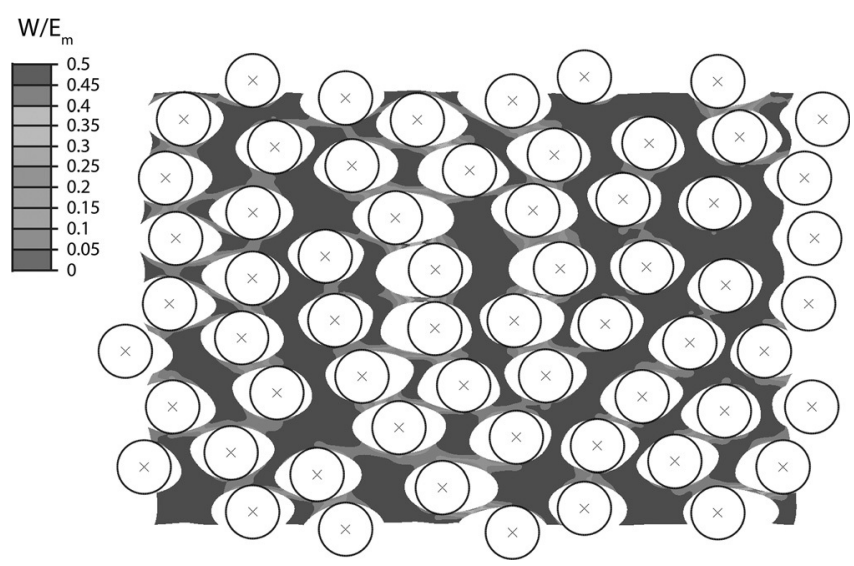

Figure 6. Microstructure and normalized elastic energy density $W$ at $36 \%$ macroscopic strain for a microstructure without interphase when debonding localization does not appear due to a high matrix stiffness $\left(E_{\mathrm{m}}=5 E_{\mathrm{m}}{ }^{0}\right)$.

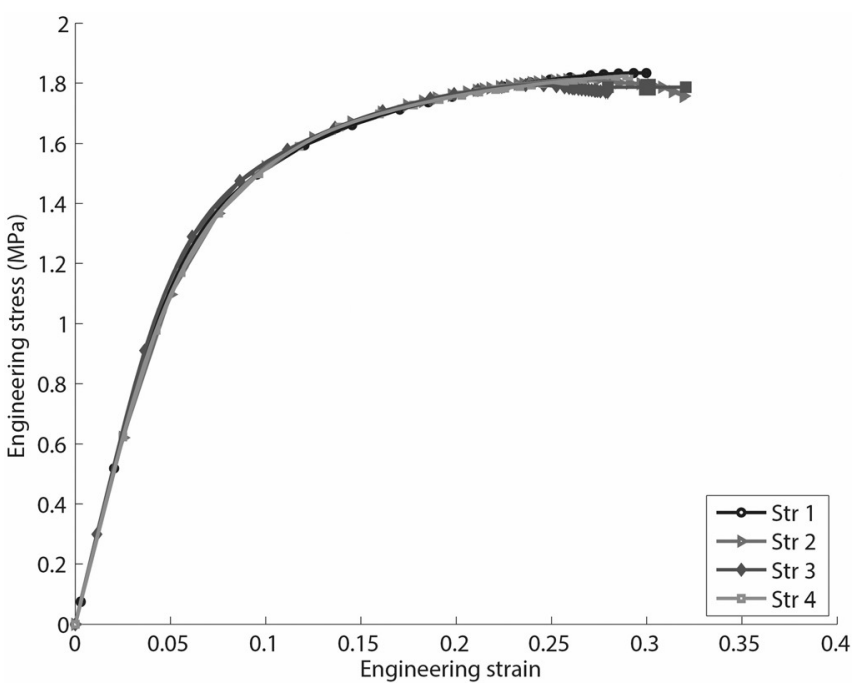

Figure 7. Uniaxial tension responses of four microstructures of identical constitutive parameters and differing by the particles layout only.

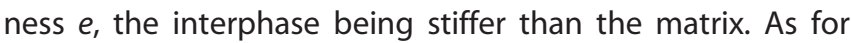
fracture, two cases can be distinguished. When the interphase is thin, the composite shows similar behavior until break as the reference presenting no interphase and a Young modulus $E_{\mathrm{m}}=E_{\mathrm{m}}{ }^{0}$. When the interphase is thick, no debonding localization occurs. In this case, the composites sustain larger strain and stress at break and display a similar evolution to the one of the reference material without interphase and a Young modulus $E_{\mathrm{m}}=H . E_{\mathrm{m}}{ }^{0}$. From a microstructural point of view, it is to notice that, the interphase percolates for the second group only. Thus, a path to transmit the load through the microstructure exists and relieves the softer matrix. Consequently the structure is stiffer and behaves very similarly to a composite with a stiff matrix without interphase.

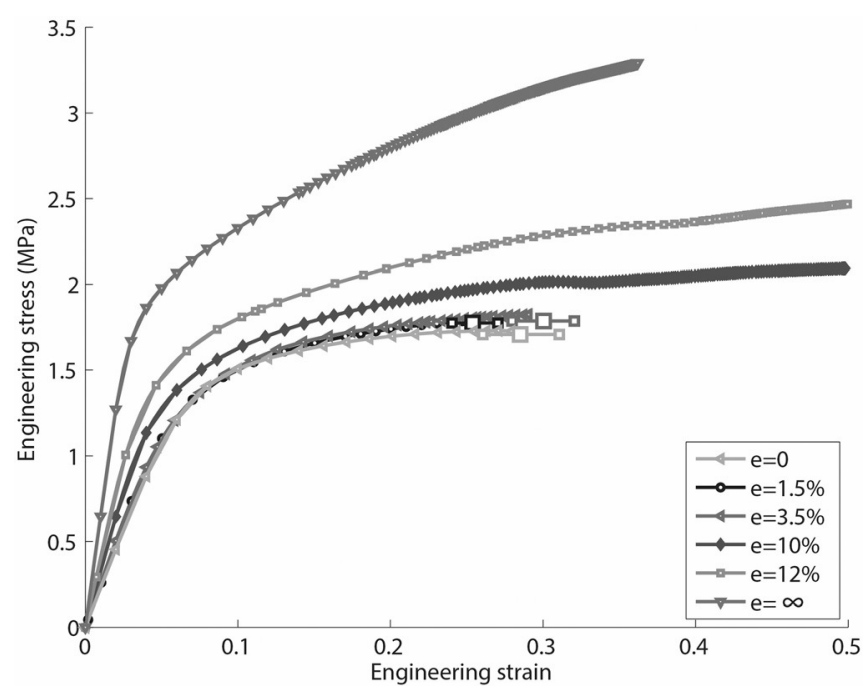

Figure 8. Effect of the interphase thickness on the uniaxial behavior of composites with interphase stiffness characterized by $H=5$. The case $e=0$ (respectively $e=\infty$ ) represents a microstructure without interphase and $E_{\mathrm{m}}=E_{\mathrm{m}}{ }^{0}$ (respectively $E_{\mathrm{m}}=H E_{\mathrm{m}}{ }^{0}$ ).

Hence, for $H=5$, the presence of a stiff interphase has a significant impact when the interphase percolates and none when it does not percolate.

According to these results, the presence of a percolating interphase enhances both stress and strain at break of the composite, which is consistent with experimental results on HTPB/AP and organic filler systems $[2,6]$ reported in the Introduction. Introducing an interphase in the simulated microstructures also leads to an increase in the initial Young modulus as observed experimentally for organic filler systems [6]. However, stress (respectively strain) at break of the numerical results for non-percolating microstructure does not show a marked increase (respectively decrease) as could be expected from experimental results. This difference could stem from the fact that on the one hand, in the calculations, the presence of a bonding agent was solely accounted for through the introduction of an interphase but no modification of the filler/matrix properties was implemented. Whereas, on the other hand, bonding agent are designed to reinforce the matrix/filler bond [41]. Yet, it has been demonstrated $[8,28]$ that a change of the critical strength or of the debonding energy of the cohesive zone, which both represent a modification of the interface, can produce such an increase in the stress at break.

\section{Effect of the Interphase Stiffness}

Figure 9 illustrates the influence of the $H$ ratio on the beneficial effect of interphase percolation on the failure of the composite. Two interphase relative thicknesses with $(e=$ $0.12)$ and without $(e=0.035)$ percolation were tested, each with two values of the ratio $H, 2$ and 10, compared to the reference case $H=5$. For a thin interphase (Figure 9a), the 


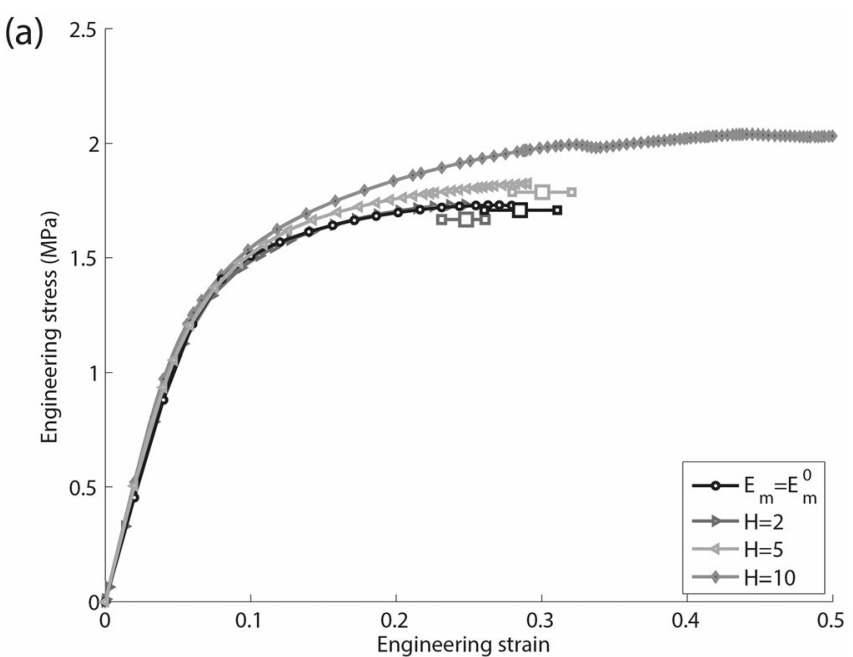

(b)

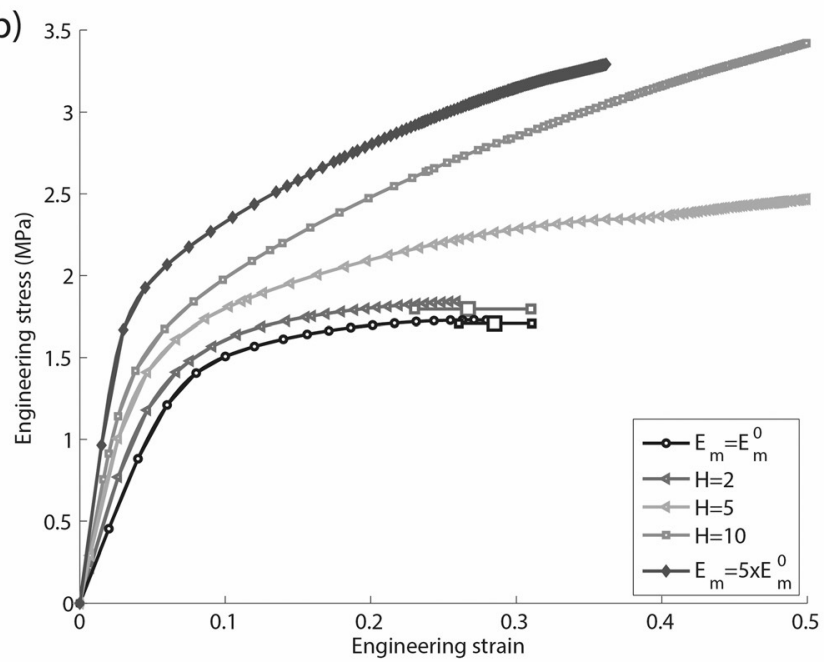

Figure 9. Comparison of the uniaxial behaviors of microstructures for different rigidity ratios $H$ with fixed interphase relative thickness: (a) $e=0.035$, (b) $e=0.12$. In the cases $E_{\mathrm{m}}=E_{\mathrm{m}}{ }^{0}$ (respectively $\left.E_{\mathrm{m}}=5 E_{\mathrm{m}}{ }^{0}\right)$, there is no interphase but a matrix with stiffness $E_{\mathrm{m}}{ }^{0}$ (respectively $5 E_{\mathrm{m}}{ }^{\circ}$ ) only.

stiffer the interphase, the stiffer the composite and the larger its stress at failure. As regards the strain at failure, at low values of ratio $H$, it is similar to the reference case $E_{\mathrm{m}}=$ $E_{\mathrm{m}}{ }^{0}$ : the failure remains unaffected by the presence of the interphase. At a higher value of ratio $H$ the strain at break increases significantly.

For a percolated interphase (Figure 9b), the stiffer the interphase, the stiffer the composite at small strain. Concerning the failure properties, two cases appear: (i) for stiff interphases $(H=5$ and 10$)$ a $50 \%$ increase of the stress and strain at break is achieved, whereas (ii) for a soft interphase $(H=2)$ no noticeable influence on the failure behavior is observed. Note that for this relative thickness $e$ and a sufficiently high value of the $H$ ratio, the mechanisms are similar to the one observed for the reference case without interphase and high matrix stiffness.
According to these results, the presence of a percolating interphase is not always sufficient to achieve an improvement of both stress and strain at break, a sufficiently stiff interphase in comparison to the matrix is also necessary. Besides, a non-percolated interphase can also yield improvement of the failure strain but not of the failure stress. Very local measurements such as AFM peak force are required to check these conditions and they cannot be easily predicted when formulating the material. For these reasons the presence of an interphase can have a range of effects on the stress and strain at break - improving both or not affecting them - that cannot be interpreted without local measurements. This could explain the discrepant experimental results found in the literature and the difficulties experienced to consistently interpret them.

\section{Conclusions}

AFM observations with Young modulus measurements at the microscale were conducted. The presence of an interphase between matrix and fillers in industrial solid propellants was experimentally observed and its morphology and Young modulus were evaluated. An interesting feature of this interphase is to possibly percolate through the matrix. So as to understand the effect of this interphase on the mechanical behavior, a propellant-like highly filled elastomer was modeled by $2 \mathrm{D}$ periodic cells containing randomly dispersed rigid particles coated by an elastomer interphase and embedded in an elastomer matrix. Debonding of the interphase/filler interface was permitted through the use of a cohesive zone model. A failure criterion was proposed as the appearance of a fibrillar microstructure and two constitutive parameters of the interphase were varied: the relative thickness of the interphase $e$ and the ratio $H$ of interphase stiffness over matrix stiffness. Changing e revealed the significant impact of the percolation of the interphase by reducing the localization of filler/matrix debonding and therefore increasing the stress and strain at failure.

Nonetheless, as variations of $H$ have shown, conditions on the interphase stiffness are preponderant. At low interphase stiffness, there is no room for improvement of failure properties. At medium interphase stiffness, the interphase percolation is a necessary condition to obtain an increase of both strain and stress at failure. At high interphase stiffness, the failure strain can be improved without percolation but it is required to enhance the failure stress.

According to these mechanisms, adding bonding agents to propellants formulations can have a favorable impact on both stress and strain at failure in two cases: for a stiff percolating interphase, and in a more limited respect, for a very stiff interphase. In the other cases, no significant impact is observed if it is assumed that the interface properties are unchanged. These conditions on the interphase are very local properties that are difficult to predict precisely a priori. This can explain the discrepant results obtained 
by authors when evaluating the effect of a given bonding agent on the mechanical response.

\section{Acknowledgments}

The authors wish to thank DGA for its financial support.

\section{References}

[1] A. Davenas in: Technologie des Propergols Solides, Masson, Paris, 1988.

[2] H. Allen, Composite Solid Propellant with Additive to Improve the Mechanical Properties Thereof, US Patent 3745074, The United States of America as represented by the Secretary of the Army, Decatur, AL, USA, 1973.

[3] J.P. Consaga, Bonding Agent for Composite Propellants, US Patent 4944815, The United States of America as represented by the Secretary of the Navy, Washington, D.C., USA, 1980.

[4] C. S. Kim, Filler Reinforcement of Polyurethane Binder Using a Neutral Polymeric Bonding Agent, US Patent 4915755, Sacramento, CA, USA, 1990.

[5] A. E. Oberth, R. S. Bruenner, Tear Phenomena around Solid Inclusions in Castable Elastomers, Trans. Soc. Rheol. 1965, 9, 165-186.

[6] C. S. Kim, P. N. Noble, C. H. Zoun, D. Tarrant, A. Gao, The Mechanism of Filler Reinforcement from Addition of Neutral Polymeric Bonding Agents to Energetic Polar Propellants, Propellants Explos. Pyrotech. 1992, 17, 51-58.

[7] E. Landsem, T. L. Jensen, F. K. Hansen, E. Unneberg, T. E. Kristensen, Neutral Polymeric Bonding Agents (NPBA) and Their Use in Smokeless Composite Rocket Propellants Based on HMX-GAP-BuNENA, Propellants Explos. Pyrotech. 2012, 37, 581-591.

[8] P.-A. Toulemonde, J. Diani, P. Gilormini, N. Desgardin, On the Account of a Cohesive Interface for Modeling the Behavior Until Break of Highly Filled Elastomers, Mech. Mater. 2016, 93, 124-133.

[9] S.-L. Gao, E. Mader, Characterisation of Interphase Nanoscale Property Variations in Glass Fibre Reinforced Polypropylene and Epoxy Resin Composites, Composites Part A 2002, 33, 559-576.

[10] S.-H. Lee, S. Wang, G. M. Pharr, H. Xu, Evaluation of Interphase Properties in a Cellulose Fiber-Reinforced Polypropylene Composite by Nanoindentation and Finite Element Analysis, Composites Part A 2007, 38, 1517-1524.

[11] B. V. Derjaguin, V. M. Muller, Yu. P. Toporov, Effect of Contact Deformations on the Adhesion of Particles, J. Colloid Interface Sci. 1975, 53, 314-326.

[12] A. Pakzad, J. Simonsen, R. S. Yassar, Gradient of Nanomechanical Properties in the Interphase of Cellulose Nanocrystal Composites, Compos. Sci. Technol. 2012, 72, 314-319.

[13] Y. Wang, T. H. Hahn, AFM Characterization of the Interfacial Properties of Carbon Fiber Reinforced Polymer Composites Subjected to Hygrothermal Treatments, Compos. Sci. Technol. 2007, 67, 92-101.

[14] T. J. Young, M. A. Monclus, T. L. Burnett, W. R. Broughton, S. L. Ogin, P. A. Smith, The Use of the PeakForce Quantitative Nanomechanical Mapping AFM-Based Method for High-Resolution Young's Modulus Measurement of Polymers, Meas. Sci. Technol. 2011, 22, 125703.
[15] F. T. Fisher, L. C. Brinson, Viscoelastic Interphases in PolymerMatrix Composites: Theoretical Models and Finite-Element Analysis, Compos. Sci. Technol. 2001, 61, 731-748.

[16] W. Wang, K. Sadeghipour, G. Baran, Finite Element Analysis of the Effect of an Interphase on Toughening of a Particle Reinforced Polymer Composite, Composites Part A 2008, 39, 956964.

[17] D. W. Spring, G. H. Paulino, Computational Homogenization of the Debonding of Particle Reinforced Composites: The Role of Interphases in Interfaces, Comput. Mater. Sci. 2015, 109, 209224.

[18] C. D. Bencher, R. H. Dauskardt, R. O. Ritchie, Microstructural Damage and Fracture Processes in a Composite Solid Rocket Propellant, J. Spacecrafts Rockets 1995, 32, 328-334.

[19] Z. J. Tao, S. D. Ping, Z. Mei, Z. P. Cheng, 12th International Symposium on Multiscale, Multifunctional and Functionally Graded Materials, Beijing, P. R. China, October 22-26, 2013.

[20] L. A. Vratsanos, R. J. Farris, A Predictive Model for the Mechanical Behavior of Particulate Composites. Part II: Comparison of Model Predictions to Literature Data, Polym. Eng. Sci. 1993, 33, 1466-1474.

[21] H. Tan, C. Liu, Y. Huang, P. H. Geubelle, The Cohesive Law for the Particle/Matrix Interfaces in High Explosives, J. Mech. Phys. Solids 2005, 53,1892-1917.

[22] H. M. Inglis, P. H. Geubelle, K. Matous, H. Tan, Y. Huang, Cohesive Modeling of Dewetting in Particulate Composites: Micromechanics vs. Multiscale Finite Element Analysis, Mech. Mater. 2007, 39, 580-595.

[23] L. Brassart, H. M. Inglis, L. Delannay, I. Doghri, P. H. Geubelle, An Extended Mori-Tanaka Homogenization Scheme for Finite Strain Modeling of Debonding in Particle-Reinforced Elastomers, Comput. Mater. Sci. 2009, 45, 611-616.

[24] J. Segurado, J. Llorca, A New Three-Dimensional Interface Finite Element to Simulate Fracture in Composites, Int. J. Solid Struct. 2004, 41, 2977-2993.

[25] K. Matous, P. H. Geubelle, Finite Element Formulation for Modeling Particle Debonding in Reinforced Elastomers Subjected to Finite Deformations, Comput. Methods Appl. Mech. Eng. 2006, 196, 620-633.

[26] K. Matous, H. M. Inglis, X. Gu, D. Rypl, T. L. Jackson, P. H. Geubelle, Multiscale Modeling of Solid Propellants: From Particle Packing to Failure, Compos. Sci. Technol. 2007, 67, 1694-1708.

[27] X. A. Zhong, W. G. Knauss, Analysis of Interfacial Failure in Particle-Filled Elastomers, J. Eng. Mater. Technol. 1997, 119, 198204.

[28] J. Moraleda, J. Segurado, J. Llorca, Effect of Interface Fracture on Tensile Deformation of Fiber-Reinforced Elastomers, Int. J. Solid Struct. 2009, 46, 4287-4297.

[29] D. Ngo, K. Park, G. H. Paulino, Y. Huang, On the Constitutive Relation of Materials with Microstructure Using a PotentialBased Cohesive Model for Interface Interaction, Eng. Fract. Mech. 2010, 77, 1153-1174.

[30] Abaqus/Standard, Dassault Systèmes Simulia Corporation, Providence, RI, USA, 2010.

[31] S. Torquato, Random Heterogeneous Materials, Springer, Heidelberg, 2002.

[32] S. E. Leon, G. H. Paulino, A. Pereira, I. F. M. Menezes, E. N. Lages, K. Park, A Unified Library of Nonlinear Solution Schemes, Appl. Mech. Rev. 2011, 64, 040803-1-26.

[33] V. Tvergaard, J. W. Hutchinson, The Influence of Plasticity on Mixed Mode Interface Toughness, J. Mech. Phys. Solids 1993, 41, 1119-1135.

[34] A. Turon, C. G. Davila, P. P. Camanho, J. Costa, An Engineering Solution for Mesh Size Effects in the Simulation of Delamina- 
tion Using Cohesive Zone Models, Eng. Fract. Mech. 2007, 74, 1665-1682.

[35] H. Tan, Y. Huang, C. Liu, G. Ravichandran, H. M. Inglis, P. H. Geubelle, The Uniaxial Tension of Particulate Composite Materials with Nonlinear Interface Debonding, Int. J. Solid Struct. 2007, 44,1809-1822.

[36] A. Azoug, Micromécanismes et Comportement Macroscopique d'un Élastomère Fortement Chargé, PhD Thesis, Ecole Polytechnique, Paris, France, 2010.

[37] W. Yan-Qing, H. Feng-Lei, A Micromechanical Model for Predicting Combined Damage of Particles and Interface Debonding in PBX Explosives, Mech. Mater. 2009, 41, 27-47.

[38] R. S. Rivlin, A. G. Thomas, Rupture of Rubber. I. Characteristic Energy for Tearing, J. Polym. Sci. 1953, 10, 291-318.

[39] C. T. Liu, L. M. Klynn, J. D. Thompson, in Monitoring Microstructural Evolution and Crack Formation in a Solid Propellant under
Incremental Strain Condition using Digital Radiography X-ray Techniques, Technical Report, Defense Technical Information Center, ADA423473, Fort Belvoir, VA, USA, 2004.

[40] S. Ozupek, E. B. Becker, Constitutive Modelling of High-Elongation Solid Propellants, J. Eng. Mater. Technol. 1992, 114, 111115.

[41] K. Hori, A. Iwama, T. Fukuda, On the Adhesion Between Hydroxyl-Terminated Polybutadiene Fuel-Binder and Ammonium Perchlorate. Performance of Bonding Agents, Propellants Explos. Pyrotech. 1985, 10, 176-180.

Received: March 17, 2016 Published online: August 25, 2016 